\title{
Evidence for negative charging of the lunar surface in shadow
}

\author{
J. S. Halekas, ${ }^{1}$ D. L. Mitchell, ${ }^{1}$ R. P. Lin, ${ }^{1}$ L. L. Hood, ${ }^{2}$ M. H. Acuña, ${ }^{3}$ \\ and A. B. Binder ${ }^{4}$ \\ Received 21 November 2001; revised 2 January 2002; accepted 9 January 2002; published 25 May 2002.
}

[1] Observations of electron distributions above the shadowed surface of the Moon show energy-dependent loss cones which indicate reflection by both magnetic and electric fields. At the same time, low energy $(\$ 100 \mathrm{eV})$ field aligned upward-going electron beams are observed. Together, these observations imply average night-side potential differences between the surface and the Lunar Prospector (LP) spacecraft of $\sim-35 \mathrm{~V}$. The lunar surface may be at an even higher negative potential relative to the ambient plasma, since LP will likely also charge negative. The potential difference is consistent with simple current balance models which include secondary emission. No clear dependence is found on surface terrane type and age, or on ambient electron density and temperature. Instead, the potential difference is found to depend strongly on the angle from the subsolar point and the angle between the magnetic field and the normal to the lunar surface. INDEX TERMS: 5421 Planetology: Solid Surface Planets: Interactions with particles and fields; 5470 Planetology: Solid Surface Planets: Surface materials and properties; 6250 Planetology: Solar System Objects: Moon (1221); 7855 Space Plasma Physics: Spacecraft sheaths, wakes, charging

\section{Introduction}

[2] It has long been expected on purely theoretical grounds that the shadowed surface of the Moon should charge negative. An object in a plasma with comparable electron and ion densities and temperatures will charge negative if there is not another current source (e.g. photoemission). This is a wellknown phenomenon for spacecraft in eclipse situations [DeForest, 1972] and occurs because of the higher mobility of the much lighter electrons. However, other than suprathermal ion data from the SIDE experiment onboard Apollo 14, which implied lunar potentials of $\sim 100 \mathrm{~V}$ negative near the terminator [Lindeman et al., 1973] few measurements of lunar night side potentials exist.

[3] The Electron Reflectometer onboard LP is an electrostatic analyzer which measures the full 3-d electron distribution from 10 $\mathrm{eV}$ to $20 \mathrm{keV}$. This instrument was designed to measure the magnetic reflection of electrons from surface crustal magnetic fields [Lin et al., 1998]. In the absence of magnetic fields, ambient electrons striking the surface of the Moon will almost all be absorbed. However, magnetic anomalies ranging in magnitude from a few tenths of a nanotesla or less to hundreds of nT, and ranging in size from a few $\mathrm{km}$ or less to several hundred $\mathrm{km}$, are present over much of the lunar surface. These magnetic fields reflect some electrons before they can reach the surface. This reflection depends on the pitch angle $\alpha$ (the angle between the electron velocity and the magnetic field). Reflected electrons will

\footnotetext{
${ }^{1}$ Space Sciences Laboratory, University of California, Berkeley, USA.

${ }^{2}$ Lunar and Planetary Laboratory, University of Arizona, Tucson, USA.

${ }^{3}$ NASA Goddard Space Flight Center, Greenbelt, Maryland, USA.

${ }^{4}$ Lunar Research Institute, Tucson, Arizona, USA.
}

be seen up to a cutoff pitch angle $\alpha_{c}$, beyond which there will be a "loss cone" devoid of particles. By assuming conservation of total energy and the first adiabatic invariant (magnetic moment) one can derive that $\sin ^{2} \alpha_{c}=B_{L P} / B_{S}$, where $B_{L P}$ is the magnetic field magnitude at LP, and $B_{S}$ is the surface field. Note that this expression is independent of energy.

\section{Observations}

[4] Our observations reveal something quite different from the expected energy-independent magnetic reflection. Figure 1 shows a typical measurement of differential electron energy flux versus pitch angle and energy. A loss cone around $180^{\circ}$ is clearly seen at energies from $\sim 100-1000 \mathrm{eV}$. However, the cutoff pitch angle is not constant as a function of energy. Instead the angular extent of the loss cone increases with energy. Furthermore, at the lowest energies the loss cone is no longer visible. Instead, we observe a beam of electrons travelling upward along the field line from the surface.

[5] The data is presented as differential energy flux, which is proportional to instrument counts. The very lowest fluxes are shown in dark blue, with the isolated diamonds showing single counts. The observed fluxes span three orders of magnitude, as do the instrument counts. Thus statistical errors for all but the lowest fluxes are small.

[6] Similar observations are ubiquitous. All of the data presented here are collected on the night side in the geotail or in the solar wind wake, at an altitude range of $20-40 \mathrm{~km}$. In these two environments, almost without fail, we observe similar energydependent loss cones and upward-going electron beams.

\section{Interpretation}

[7] The energy dependence of the loss cone alone makes it immediately clear that adiabatic magnetic reflection alone cannot be responsible for this distribution. However, by adding electric fields to our previous treatment, we can derive that $\sin ^{2} \alpha_{c}=$ $B_{L P} / B_{S} \times(1+e \Delta U / E)$ [Feldman et al., 1983], where $\Delta U$ is the potential difference between the surface and LP, E is the kinetic energy of the particles at LP, and $\mathrm{e}$ is the magnitude of the electron charge. We fit this expression to measurements at four energies (data for these energies has been sorted by pitch angle at high time resolution onboard LP and does not correspond precisely to that shown in Figure 1), and show the results in Figure 2. The best fit gives $\Delta U=-48 \pm 15 \mathrm{~V}$. The error is high, since we fit to a curve using only four data points with statistical errors. However, the result is inconsistent with a potential difference of zero.

[8] We can obtain more information from the upward-going beam of electrons. These electrons are present at much higher fluxes than downgoing electrons and therefore cannot be reflected primary electrons. We suggest that the beam is composed of secondary electrons which are emitted at low energies and accelerated through a potential difference as they travel from the surface to LP. Secondary emission coefficients do not greatly exceed unity, so it may seem contradictory that the beam fluxes are so much higher. However, the secondary electrons are concentrated in a much smaller energy range. We subtract a 


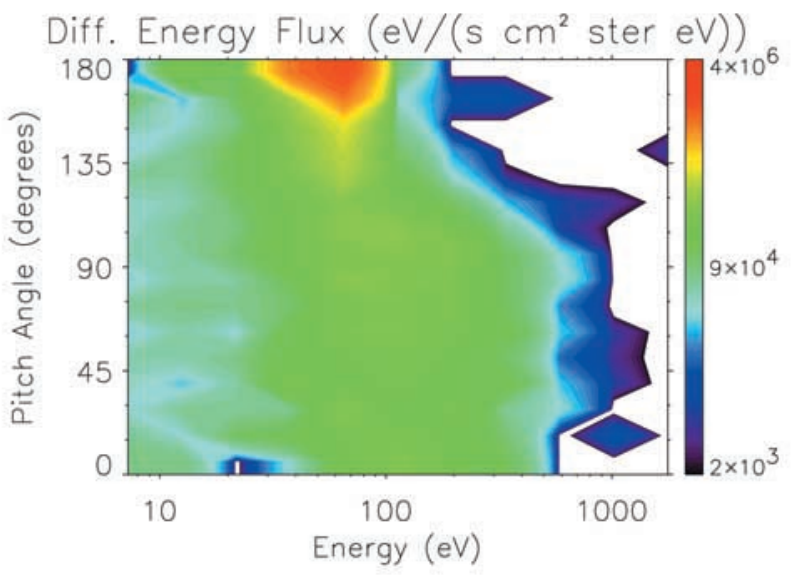

Figure 1. Differential electron energy flux (in color) versus energy and pitch angle. These measurements were made at 13:39 UT on March 31, 1999. At this time the Moon was in the Earth's geotail and LP was above the shadowed surface of the Moon.

theoretical loss cone distribution and fit the remaining beam distribution to a Gaussian. We find a center energy of $51 \mathrm{eV}$, and a FWHM of $30 \mathrm{eV}$. The center energy matches the potential difference we found from measuring the loss cone angle versus energy, supporting our hypothesis that the beam is composed of accelerated secondary electrons. The beam seems too broad, since secondaries should start with a narrow energy range of a few $\mathrm{eV}$ [Whipple, 1981]. However, this is largely explained by our energy resolution. The analyzer $\Delta E / E$ is $\sim 24 \%$, and adjacent energy steps are summed together, resulting in an effective energy width for each bin of $\Delta E / E=0.48$. Also, our measurements represent an average over the gyrodiameters of the electrons in question (several $\mathrm{km}$ ), and the surface potential may be nonuniform over these scales.

[9] Where comparisons have been performed, as above, the potential difference determined from the loss cone generally matches the center energy of the beam. We find an average potential difference of $-32 \mathrm{~V}$ in the solar wind wake, and -38 $\mathrm{V}$ in the night-side geotail.

\section{Expectations}

[10] Surface charging is a well-researched phenomenon, since spacecraft charging affects low-energy plasma measurements. A body will charge to a potential such that there is no net current to its surface. In practice, of course, this process is complex. However, it is possible to construct simple qualitative models. If both electron and ion populations are Maxwellian, unmodified current densities are given by $J_{0 s}=n q_{s} \sqrt{k T_{s} /\left(2 \pi m_{s}\right)}$. If the temperatures are similar, then the electron current is $\sqrt{m_{i} / m_{e}}$ or $\sim 40$ times greater. A surface will charge negative until these currents balance. The modified electron current density is then given by $J_{e}=J_{0 e} e^{e U / k T_{e}}$, and the ion current density is given by either $J_{i}=J_{0 i}$ (thin sheath) or $J_{i}=J_{0 i} \times\left(1-e U / k T_{i}\right)$ (thick sheath) depending on the size of the body relative to the Debye length [Whipple, 1981].

[11] The Moon is large compared to the Debye length (hundreds of meters in the wake), so the thin sheath approximation is valid. We use typical electron paramaters measured by LP in the solar wind wake of $n_{e}=0.01 \mathrm{~cm}^{-3}$ and $T_{e}=50 \mathrm{eV}$. Observations by WIND showed that ion densities were comparable to electron densities, while ion temperatures were a factor of $\sim 5$ lower [Bosqued et al., 1996; Ogilvie et al., 1996]. These measurements were taken at $\sim 6 R_{S}\left(10^{4} \mathrm{~km}\right)$ farther down the wake than our lowaltitude orbit. However, we use $n_{i}=n_{e}$ and $T_{i}=10 \mathrm{eV}$ as a first approximation. Balancing the electron and ion currents then gives $U_{\text {MOON }}=-230 \mathrm{~V}$. The LP spacecraft is exposed to the same plasma and will also charge negative. LP is small compared to the Debye length, so the thick sheath approximation is valid. This gives $U_{L P}=-104 \mathrm{~V}$, and $\Delta U=-126 \mathrm{~V}$. Unfortunately, though negative charging of spacecraft in shadow has been measured at higher electron temperatures of several $\mathrm{keV}$ [DeForest, 1972], it appears that few measurements of spacecraft potentials in similar regimes have been made.

[12] The effects of secondary emission are quite significant for the electron energies in question. Adding lunar secondary emission modifies the electron current density expression to $J_{e_{t o t}}=J_{e} \times\left(1-\delta_{\text {eff }}\right)$, where $\delta_{\text {eff }}=\left(\int \frac{d J_{e}(E)}{d E} \delta(E) d E\right) / J_{e}$. We use secondary emission characteristics measured from lunar dust for $\delta(E)$ [Horányi et al., 1998]. Assuming isotropic Maxwellian incidence, we calculate that $\delta_{\text {eff }}=0.87$. This results in $U_{M O O N}$ $=-125 \mathrm{~V}$, and $\Delta U=-21 \mathrm{~V}$.

[13] This prediction is close to the value we observe, though we have neglected secondary emission from the spacecraft, which will push $U_{L P}$ less negative and increase the magnitude of $\Delta U$. Also, our assumption of isotropic Maxwellian incidence is at best an approximation. LP data reveal a significant non-Maxwellian tail to the electron distributions. Meanwhile, the charging of the surface will ensure that electron incidence is not isotropic. However, this simple model gives results of the same order of magnitude as our measurements nonetheless. Other predictions have been made, but differing assumptions about ambient plasma parameters and secondary emission characteristics have led to estimates of lunar night-side potentials which vary from 0 to $-1800 \mathrm{~V}$ [Knott, 1973; Manka, 1973].

\section{Dependence on Surface Characteristics}

[14] We use a pre-existing data set constructed from geologic maps of the lunar near side [Halekas et al., 2001; Wilhelms and McCauley, 1971] to look for changes in the potential difference measured over different terranes. Table 1 shows the results of this survey. We find no clear differences for different types or ages of terranes. This is perhaps not surprising, since secondary emission is controlled by a very thin layer on the surface [Whipple, 1981]. This layer is well gardened by small impacts and may not vary

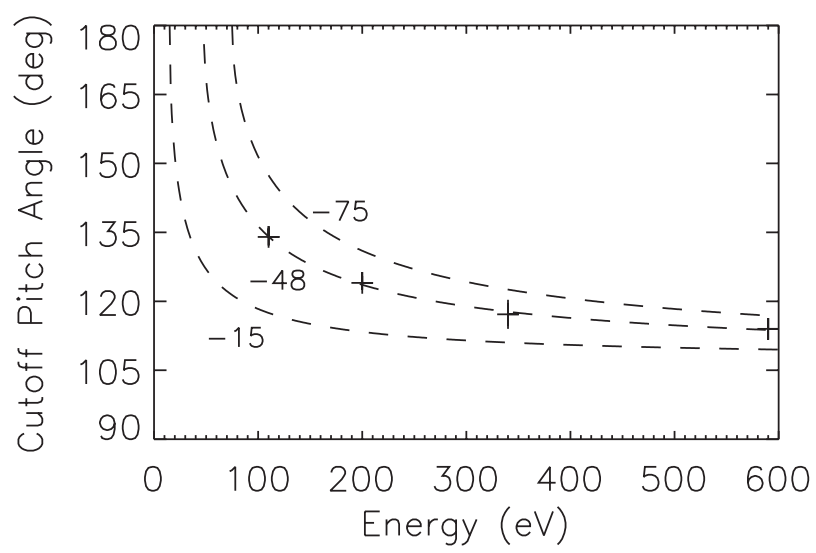

Figure 2. Loss cone angles calculated from high time resolution measurements of electron pitch angle distributions at four energies $(110,200,340$, and $590 \mathrm{eV})$, taken during the same interval as the data used in Figure 1, with error bars estimated from Monte Carlo simulations. A theoretical curve taking into account the effects of electric and magnetic reflection is fitted to the data points and shown as a dashed line. The fit gives $\Delta U=-48 \pm 15 \mathrm{~V}$, with the error again estimated from Monte Carlo simulations. Curves are also shown for $\Delta U=-15 \mathrm{~V}$ and $-75 \mathrm{~V}$ for comparison. 
Table 1. Potential Difference vs. Terrane

\begin{tabular}{lcc}
\hline \multicolumn{1}{c}{ Terrane } & Data Points & Average $\Delta U$ \\
\hline All Near Side & 7000 & -32.9 \\
Pre-Imbrian & 607 & -33.4 \\
Imbrian & 4681 & -31.9 \\
Eratosthenian & 596 & -31.7 \\
Copernican & 489 & -34.0 \\
Maria & 3255 & -31.9 \\
Craters & 1269 & -32.7 \\
Plains/Terra & 1130 & -31.9 \\
\hline
\end{tabular}

greatly in its secondary emission characteristics in different regions.

\section{Dependence on Ambient Plasma Environment and Position}

[15] To test for a correlation with ambient plasma parameters, we bin potential difference data from the solar wind wake by electron density and temperature. We usually only measure energies above $\sim 40 \mathrm{eV}$, so rather than relying on partial moments, we fit the distributions to Kappa functions [Scudder, 1992] to estimate moments. The results are shown in Figure 3. While our simple treatment made it clear that any dependence on temperature would be complicated by secondary emission, some dependence seems called for. Instead, we find an unexpected correlation with density. Unfortunately, however, the plasma parameters that we measure may be affected by spacecraft charging. If LP charges negative as we expect, we will underestimate the ambient electron density. Also, since LP measurements show the presence of a high energy non-Maxwellian tail, we may overestimate the electron temperature.

[16] We find that the potential difference correlates much more strongly with two angles. One is the angle from the subsolar point, the other the tip angle between the magnetic field and the normal to the surface. We bin the potential difference by these two angles and show the results in Figure 4. The magnitude of the potential difference decreases as either angle increases.

[17] Electron temperature and density both depend strongly on the angle from the subsolar point. The night-side density falls off

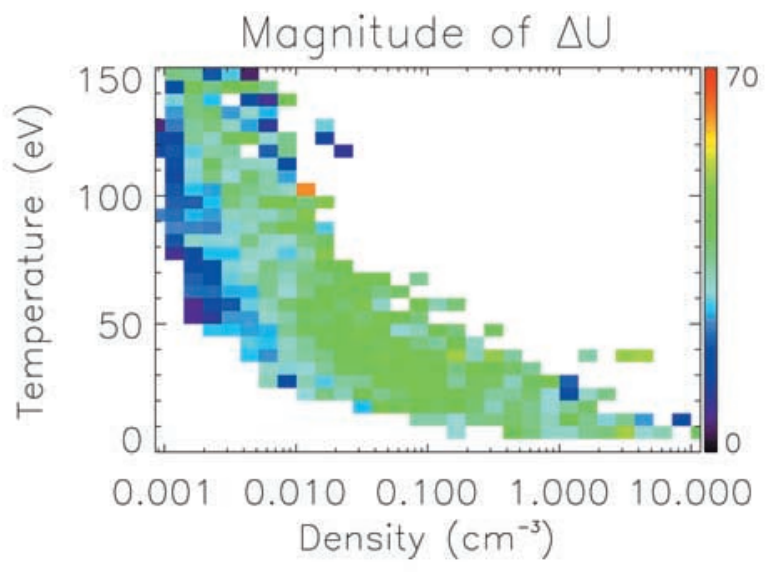

Figure 3. Potential difference measurements from the solar wind wake are binned by ambient electron density and temperature and color-coded by magnitude. The potential difference is determined from measurements of the loss cone angle at multiple energies. The electron density and temperature are obtained from Kappa function fits to the electron distributions measured by LP.

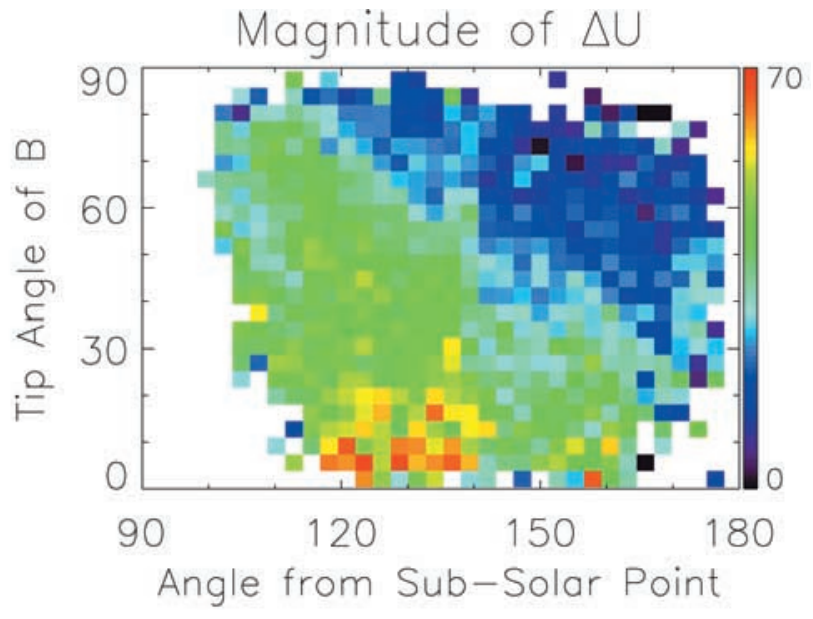

Figure 4. Potential difference measurements from the solar wind wake are binned by two angles and color-coded by magnitude. The potential difference is determined from measurements of the loss cone angle at multiple energies. The two axes are the angle from the subsolar point (in Moon-centered coordinates) and the tip angle between the magnetic field and the normal to the surface.

steeply from the terminator, and the temperature increases gradually (this explains why the data in Figure 3 lie roughly on a curve of $n$ vs. T). The observed dependence on this angle is much clearer than any observed dependence on electron density and temperature. However, it is possible that the root of the dependence lies with some property of the ambient electron distribution, especially if our measurements have been affected by the charging of LP. The dependence on tip angle requires another explanation, though. Most of the data presented here is taken over areas of weak or moderate magnetic fields, so the curvature of field lines is probably not important, and all field lines should map to the solar wind. The dependence may instead result from differences between electron and ion motion in the wake. The electrons have small gyroradii and are tightly constrained to follow the magnetic field lines, while the ions have much larger gyroradii and may have drift motions which are significant.

[18] The results of similar binning of night-side geotail data do not make the situation any clearer. The range of parameter space covered by the geotail data is much smaller, and it neither greatly clarifies nor contradicts the observed effects.

\section{Conclusions}

[19] Our data show that, when the lunar surface lies in shadow, we virtually always observe evidence of negative charging. Measurements of energy-dependent loss cones and accelerated secondary electrons give comparable estimates of the potential difference. The average inferred surface potential is $\sim-35 \mathrm{~V}$ with respect to LP. Simple current balance models which take into account secondary emission yield predictions of this order of magnitude.

[20] No clear dependence on the type or age of surface geologic terrane is found. Surprisingly, binning the data by electron density and temperature also reveals little in the way of clear trends, other than some tendency for low electron densities to be associated with lower magnitudes of the potential difference. Clear anticorrelations are found, however, between potential difference magnitude and both the angle from the subsolar point and the tip angle of the magnetic field.

[21] Acknowledgments. Research at the University of California, Berkeley, was supported by NASA through subcontract LRI-99-101 from 
the Lunar Research Institute. We thank two anonymous referees for helpful and constructive reviews.

\section{References}

Bosqued, J. M., N. Lormant, H. Reme, C. d'Uston, R. P. Lin, K. A Anderson, C. W. Carlson, R. E. Ergun, D. Larson, J. McFadden, M. P. McCarthy, G. K. Parks, T. R. Sanderson, and K. P. Wenzel, Moon-solar wind interaction: First results from the WIND/3DP experiment, Geophys. Res. Lett., 23, 1259-1262, 1996.

DeForest, S. E., Spacecraft charging at synchronous orbit, J. Geophys. Res, 77, 651-659, 1972.

Feldman, W. C., R. C. Anderson, S. J. Bame, S. P. Gary, J. T. Gosling, D. J McComas, and M. F. Thomsen, J. Geophys. Res, 96, 96-110, 1983.

Halekas, J. S., D. L. Mitchell, R. P. Lin, S. Frey, L. L. Hood, M. H. Acuña and A. B. Binder, Mapping of crustal magnetic anomalies on the lunar near side by the Lunar Prospector electron reflectometer, J. Geophys. Res., 106, 27,841-27,852, 2001.

Horányi, M., B. Walch, S. Robertson, and D. Alexander, Electrostatic charging properties of Apollo 17 lunar dust, J. Geophys. Res., 103, 85758580, 1998.

Knott, K., Electrostatic charging of the lunar surface and possible consequences, J. Geophys. Res., 78, 3172-3175, 1973.

Lin, R. P., D. L. Mitchell, D. W. Curtis, K. A. Anderson, C. W. Carlson, J. McFadden, M. H. Acuña, L. L. Hood, and A. Binder, Lunar surface magnetic fields and their interaction with the solar wind: Results from Lunar Prospector, Science, 281, 1480-1484, 1998.
Lindeman, R., J. W. Freeman, and R. R. Vondrak, Ions from the lunar atmosphere, Proc. 4th. Lunar Science Conf., 2889-2896, 1973.

Manka, R. H., Plasma and potential at the lunar surface, in Photon and Particle Interactions with Surfaces in Space, edited by R. J. L. Grard and D. Reidel, pp. 347-361, Dordrecht, Netherlands, 1973.

Ogilvie, K. W., J. T. Steinberg, R. J. Fitzenreiter, C. J. Owen, A. J. Lazarus, W. M. Farrell, and R. B. Torbert, Observations of the lunar plasma wake from the WIND spacecraft on December 27, 1994., Geophys. Res. Lett., 23, 1255-1258, 1996.

Scudder, J. D., On the causes of temperature change in inhomogeneous low-density astrophysical plasmas, Astrophys. J., 398, 299-318, 1992.

Whipple, E. C., Potentials of surfaces in space, Rep. Prog. Phys., 44, $1197-$ $1250,1981$.

Wilhelms, D. E., and J. F. McCauley, Geologic map of the near side of the Moon, U.S. Geol. Surv. Misc. Geol. Inv. Map, I-703, 1971.

J. S. Halekas, D. L. Mitchell, and R. P. Lin, Space Sciences Laboratory, University of California, Berkeley, CA 94720, USA. (jazzman@ssl.berkeley. edu)

L. L. Hood, Lunar and Planetary Laboratory, University of Arizona, Tucson, AZ 85721, USA.

M. H. Acuña, NASA Goddard Space Flight Center, Greenbelt, MD 20771, USA.

A. B. Binder, Lunar Research Institute, Tucson, AZ 85747, USA 